\title{
Simulation of laser plasma emission characteristics of small solid particles in different gas atmospheres at various pressures
}

\author{
Non-member Alexander Andreev (Institute of Laser Physics, Russia ) \\ Member Toshitsugu Ueda (Yokogawa Electric Corporation)
}

\begin{abstract}
The problem of small solid particle size and material detection is important for industrial and environmental applications. Previous investigations have shown the possibility of using the laser breakdown method to address the first issue; the sensitivity of this method is a thousand times higher than that of conventional methods. However, for small particle sizes, the damage threshold of the solid target in this case is very close to the breakdown point of pure gas. After breakdown, there is a small volume of dense hot plasma that emits radiation. We analyzed this radiation especially at the late stage using an analytical model and simulation code, and found that the emission intensity varied depending on the laser type and plasma parameters including initial particle size.
\end{abstract}

Keywards: laser pulse, small solid particle size, laser breakdown in gas, laser plasma line emission

\section{Introduction}

Laser-induced breakdown (LIB) of gas has been the subject of a number of studies for plasma generation. It has been observed in many studies that the threshold for generating shielding plasma in the presence of a solid is significantly lower than the threshold for clean gases (see for example $[1,2]$ ). First, the dependence of aerosol breakdown on its size was predicted [1] and then developed [3,4]. The particle breakdown threshold value is greatly influenced by particle material, particle diameter, laser wavelength, intensity and pulse duration.

The problem of small solid particle (SSP) size detection is important for industrial and environmental applications. Previous investigations have shown the possibility of using the laser breakdown method for such detection. The sensitivity of this method is a thousand times higher than that of conventional methods such as ICP and MIT [5]. However, for a particle size of less than $0.1 \mu \mathrm{m}$, the damage threshold of the solid target in this case is very close to the breakdown point of pure gas. After breakdown, there is a small volume of dense hot plasma that emits radiation in lines and continuum. This study set out to determine the threshold intensity required to generate dense plasma when interacting with SSP suspended in gas and its emission. We analyzed this radiation using an analytical model and simulation code as well, and found that the emission intensity varied depending on the laser type and plasma parameters including initial particle size.

\section{Computer code and numerical simulations}

The developed simulation code includes the following physical processes: propagation of a heat wave inside matter, hydrodynamics of gas atmosphere and plasma by two-temperature approximation, thermo heating, absorption of laser radiation, thermal radiation emission, and absorption by gas and plasma.

At first stage of the simulation we supposed that absorbed laser energy uniform distributed in vapour volume at initial time $t=0$. At $t>0$ we consider that heated vapor matter expands in the surrounding gas together with thermal radiation of this matter. The task is solved using a two-temperature one-fluid hydrodynamic approximation. We consider that gas matter consists of two subsystems: the subsystem of heavy particles (molecules, atoms and ions), and the electron-oscillator subsystem which consists of thermal energy of electrons, molecular energy of oscillation and dissociation, and energy of electron excitation and ionization of atoms and ions. We suppose that at any moment each subsystem is in equilibrium determined by it own temperature ( $T_{\mathrm{e}}$ for electrons and $T_{\mathrm{i}}$ for other particles) and gas density $\rho$. The energy of heavy particles is the energy of movement and rotation of components according to $T_{\mathrm{i}}$. The systems change by energy through collisions of electrons with heavy particles and between movement and rotation channels. We also take into account electron thermoconductivity and energy transformation by radiation emission and absorption of the electron oscillation subsystem. In this case our gas is in thermodynamic equilibrium at $T_{\mathrm{e}}$ and $\rho$. This permits us to use table data for equilibrium thermodynamic and optical properties of the gas [12]. We solve the problem by using the following set of equations:

$$
\begin{aligned}
& \rho r^{2} \frac{\partial r}{\partial m}=-1, \quad \frac{\partial u}{\partial t}=-r^{2} \frac{\partial\left(p+p_{e}\right)}{\partial m}, \\
& \frac{\partial U_{e}}{\partial t}+p_{e} \frac{\partial}{\partial m}\left(r^{2} u\right)+\frac{\partial}{\partial m}\left(F_{e}+F_{r}\right)=-Q_{e}-Q_{v}, \\
& \frac{\partial U}{\partial t}+p \frac{\partial}{\partial m}\left(r^{2} u\right)=Q_{e}+Q_{v}, F_{e}=k_{e} r^{2} \frac{\partial T_{e}}{\partial r}, \\
& Q_{e}=\frac{1}{\rho} \sum_{i} \frac{3 v_{e i} n_{e} m_{e} k\left(T_{e}-T\right)}{M_{i}}, \\
& Q_{v}=\frac{1}{\rho} \sum_{v} \frac{n_{v} \varepsilon_{v}}{\tau_{v}} \\
& \left(\begin{array}{l}
{\left[\exp \left(\varepsilon_{v} / k T_{e}\right)-1\right]} \\
{\left[\exp \left(\varepsilon_{v} / k T\right)-1\right]}
\end{array}\right) \\
& U=\frac{3}{2} \frac{k T}{\rho} \sum_{i} n_{i}+\frac{k T}{2 \rho} \sum_{m} n_{m} g_{m}, \\
& p=k T \sum_{i} n_{i}, p_{e}=k T_{e} n_{e}, \\
& U_{e}\left(T_{e}, \rho\right), \quad v_{\mathrm{ej}}\left(\mathrm{T}_{\mathrm{e}}, \rho\right), k_{e}\left(T_{e}, \rho\right), \\
& \tau_{\mathrm{v}}\left(\varepsilon_{\mathrm{v}}, T, T_{e}, \rho\right), n_{e}\left(T_{e}, \rho\right), n_{j}\left(T_{e}, \rho\right), \mathrm{j}=\mathrm{i}, \mathrm{m}, \mathrm{v} .
\end{aligned}
$$


where, $t$ is time, $m$ is Lagrange mass coordinate, $m \leq 0$ is gas, $m>$ 0 is initial matter, $r$ is radius, $\rho$ is density, $u$ is velocity, $U_{e}$ is energy of electron oscillated subsystem per gas mass unit, $\mathrm{T}_{\mathrm{e}}$ is electron oscillated temperature, $\mathrm{U}$ and $\mathrm{T}$ is thermal energy and temperature of heavy gas particle subsystem, $\mathrm{p}$ and $\mathrm{p}_{\mathrm{e}}$ is pressure, $F_{e}$ and $F_{r}$ are energy flows of electron conductivity and thermal radiation, $k_{c}$ is coefficient of electron conductivity, $Q_{c}$ is energy change velocity between subsystem at elastic collisions of gas particles, $Q_{v}$ is energy change velocity between subsystems at oscillation relaxation, $n_{c}$ is concentration of free electrons, $n_{j}$ is concentration of $j$-sort particles, $v_{e i}$ is elastic collision frequency of electrons with i-sort particles, $M_{i}$ and $m_{e}$ is mass of $i$-sort particles and mass of electron, $g_{m}$ is number of rotation rate per $m$-sort molecule, $n_{\mathrm{v}}$ is concentration of oscillators at energy of oscillation quanta $\varepsilon_{v}, \tau_{v}$ is time of oscillation relaxation, and $\mathrm{k}$ is Boltzmann constant. Non-equilibrium thermal radiation is given by:

$$
\frac{\partial I_{\varepsilon}^{ \pm}}{\partial s}=K_{\varepsilon}\left(I_{\varepsilon p}-I_{\varepsilon}^{ \pm}\right)
$$

where, $d s$ is length element along ray, $I_{\varepsilon}^{ \pm}(\varepsilon, \varphi, \mathbf{r}, \mathbf{t})$ is radiation intensity for photons with energy $\varepsilon$ in positive $(+)$ and negative $(-)$ directions of axis $m, \varphi$ is angle between ray and radius $r, \mu=\cos \varphi$ $\geq 0, I_{\mathrm{sp}}\left(\mathrm{T}_{\mathrm{e}}\right)$ is equilibrium radiation intensity, and $K_{\varepsilon}\left(\mathrm{T}_{\mathrm{e}}, \rho\right)$ is spectrum absorption coefficient. We calculated $K_{\varepsilon}\left(T_{e}, \rho\right)$ taking into account the radiation processes of photo-ionization, classical and line absorption. Wave functions of ground and excited states were calculated by the Hartree-Fok method. We used a semiempirical method to calculate the photo-ionization cross-section of excited states. The radiation transition cross-section was obtained with the help of calculated wave functions (see Fig. 1).

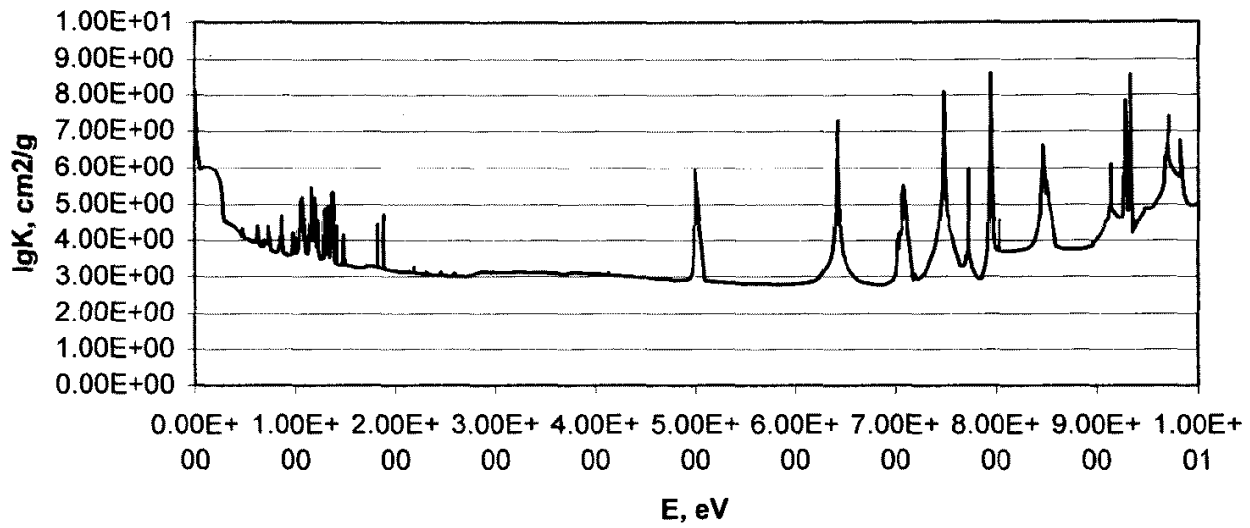

Fig. 1. Spectrum absorption coefficient

Flow $F_{\mathrm{r}}$ and flow density $\mathrm{q}_{\mathrm{r}}$ of thermal radiation represented by spectral intensity $I_{\varepsilon}^{ \pm}$are given by:

$$
\begin{array}{r}
q_{r}{ }^{ \pm}=2 \pi \int_{0}^{\infty} d \varepsilon \int_{0}^{l} I_{\varepsilon}{ }^{ \pm} \mu d \mu, \\
q_{r}=q_{r}{ }^{+}-q_{r}{ }^{-}, \quad F_{r}=r^{2} q_{r} .
\end{array}
$$

Equations describe gas movement and transfer of radiation before and after the shock wave front.

If dissociation and ionization processes do not finish in the heated layer before the shock wave, these processes can be in the front at heavy particle collisions between each other. We approximately describe these processes according to reactions with the participation of molecules and atoms of $\mathrm{O}$ and $\mathrm{N}$ in air as follows: reaction does not begin if temperature $T$ is less than $(2 / 3) \mathrm{E}_{\mathrm{k}}$, where $\mathrm{E}_{\mathrm{k}}$ is threshold energy of $\mathrm{k}$-sort reaction; the reaction begins "immediately" if $T=(2 / 3) \mathrm{E}_{\mathbf{k}}$, and in this process the energy of the electron oscillation subsystem increases by a value equal to the energy spent by the heavy particle subsystem for dissociation and ionization. The following initial conditions are used: we give density $\rho_{c}$ and radius of initially heated vapor volume $r_{c}$; at $0 \leq r \leq r_{c}$ we have $u=0, \rho=\rho_{c}$, and $T=T_{\mathrm{e}}=T_{\mathrm{c}}$, where $T_{\mathrm{c}}$ is initial temperature according to the energy absorbed; at $>r_{c}$ we have unperturbed gas at density $\rho=\rho_{\mathrm{a} 0}$, temperature $T=T_{\mathrm{e}}=T_{\mathrm{a} 0}$ and velocity $\mathbf{u}=0$. We use the following boundary conditions: at $\mathbf{r}$ $=0$, we have $\mathrm{u}=0$ and $I_{\mathrm{E}}{ }^{-}=I_{\mathrm{E}}{ }^{+}$; at $\mathrm{r}=\propto$, we have $\rho=\rho_{\mathrm{a} 0}, \mathrm{~T}=\mathrm{T}_{\mathrm{e}}=\mathrm{T}_{\mathrm{a} 0}, \mathrm{u}=0$, and $I_{\varepsilon}^{+}=I_{\varepsilon p}\left(T_{a 0}\right)$

Additionally we include the processes of vaporization of SSP and absorption of laser radiation. The vaporization wave is given by:

$$
\begin{aligned}
& \rho_{c} \cdot u_{c}=\rho_{w} \cdot\left(u_{c}+u_{w}\right)=D_{w}, \\
& q_{w}=D_{w} \cdot\left(Q_{v}+H_{w}+\frac{\left(u_{c}+u_{w}\right)^{2}}{2}\right), \\
& p_{w}=p_{v}\left(T_{w}\right), \\
& \frac{d m_{w}}{d t}=r_{w} \cdot D_{w}, \frac{d r_{c}}{d t}=-u_{c}, \quad r_{c}=r_{w},
\end{aligned}
$$

where, $Q_{v}$ is vaporization energy, $p_{v}$ is vapor pressure, $\mathrm{q}_{w}$ is intensity at vaporization wave, $\mathrm{u}_{\mathrm{c}}$ is velocity of vaporization wave, $\rho_{c}$ is solid density, $u_{w}, \rho_{w}, T_{w}, p_{w}$, $H_{w}$ are parameters after vaporization wave, $D_{w}$ is mass flow density $m_{w}$ is vapor mass, $r_{w}$ is the coordinate of wave, $r_{c}$ is $S S P$ radius, and $u_{w}<0$. 
These equations at a vaporization wave are the boundary (at $m=m_{w}$ ) for the set of gas dynamic equations, and describe self-consistent propagation of the vaporization wave and gas. For Juge conditions, $u_{c}$ $+u_{w}=c_{w}$, where $c_{w}$ is sound velocity behind the vaporization wave.

The transport of laser radiation outside and inside the focal volume is given by:

$\frac{\partial F_{L}^{\mp}}{\partial r}= \pm k_{L} \cdot F_{L}^{\mp}, \quad F_{L}^{\mp}=r^{2} \cdot q_{L}^{\mp}$

at $r \geq r_{f}$ is the radius of the focal spot.

$$
\frac{\partial{q_{L}}^{\mp}}{\partial r}= \pm k_{L} \cdot q_{L}^{\mp} \quad \text { at } \quad \mathbf{r}<\mathbf{r}_{\mathrm{f}}
$$

where, $r$ is radius, $\mathrm{F}_{\mathrm{L}}^{\mp}$ and $\mathrm{q}_{\mathrm{L}}^{\mp}$ is flow and intensity of laser radiation in the negative (-) and positive $(+)$ direction of axis $r, k_{L}\left(\rho, T_{e}\right)$ is absorption coefficient, $\rho$ is density, and $T_{c}$ is electron temperature. At the critical point $\left(\hbar \omega_{p}=\varepsilon_{L}, \omega_{p}\right.$ is electron plasma frequency, $\varepsilon_{\mathrm{L}}$ is laser quanta energy) $q_{L}{ }^{+}=c_{L} \cdot q_{L}{ }^{-}$, where $c_{L}$ is reflection coefficient.

Boundary condition: $F_{L}{ }^{-}=r_{f}^{2} \cdot q_{L f}$ at $r=+\infty, q_{L f}(t)$ is laser intensity in focus without absorption, $t$ is time. The numerical solution follows the method of $[7,8]$.

\section{Breakdown of SSP in gas atmosphere}

In the paper [9] we considered the main processes of SSP LIB. The first step of laser pulse interaction with SSP is particle heating and evaporation by laser radiation. For a small spherical particle with radius $r$ we obtained the laser intensity required to heat and vaporize this particle. The breakdown threshold laser intensity is given by [9]

$$
I_{b d} \approx \mu \frac{\omega^{2}+v_{n}^{2}}{\omega^{2}} \frac{J_{a}}{m c^{2}}\left(\frac{40}{\tau_{p} v_{n}}+\beta \frac{D}{r^{2} v_{n}}\right),
$$

for C SSP in air and $\tau_{\mathrm{p}}=10 \mathrm{~ns}$

$$
I_{b d} \approx 210^{10}\left(\frac{\lambda_{N d}}{r}\right)^{2}\left[\frac{W}{\mathrm{~cm}^{2}}\right]
$$

where, $\mu=10^{18} \mathrm{~W} / \mathrm{cm}^{2}, \tau_{\mathrm{p}}$ is laser pulse duration, $J_{a}$ is ionization potential, $v_{n}$ is collision frequency and $\beta \geq$ 1.

As our particle is located in gas, we cannot exceed the breakdown threshold of pure gas. We checked this formula by our and other experimental data. The experiment was done using PSL particles located in air, but simulation of the values was calculated using carbon particles and we obtained coexistence between experimental and theoretical results [9].

\section{Particle plasma absorption}

In the analytical model of laser pulse interaction with a small sphere of dense plasma, we use the following assumptions.

The initial plasma temperature is taken from the evaporation model [6] where $T=$ const $(r)$ inside plasma particle (PP) because the time of electron thermal wave propagation is small compared to other characteristic time scales; the Debye radius is small compared to other characteristic lengths; initial density is determined from the vapor of our model, and the density inside PP is homogeneous during laser heating and expansion.

During laser breakdown (and after if the duration of the laser pulse is longer than the breakdown time), the electron concentration in the particle vapor rises rapidly and at some point in time the plasma frequency can reach and even exceed the laser frequency at some distance from the particle center (at $\omega_{\mathrm{pe}}=\omega, n_{\mathrm{e}}=n_{\mathrm{c}}$ ); at $n_{e} / n_{c}=3$ there is a resonant point and the absorption coefficient increases greatly at $v / \omega$ $<1$. The maximum absorption coefficient is given by $3 k^{r} /(v / \omega)$.

We consider absorption of laser intensity $I_{L}$ by a small plasma sphere with volume $V$ from hydrodynamic equations. We calculated this system very roughly, supposing $r \approx r_{0}+c_{s} t$ and sound velocity $c_{\mathrm{s}}=\left(3 \mathrm{Z} T_{\mathrm{e}} / \mathrm{m}_{\mathrm{i}}\right)^{1 / 2}$, and obtained the following expression for temperature:

$$
\begin{aligned}
& T_{e} \approx 0.3 \frac{1}{N_{e}}\left(\frac{r}{r_{0}}\right)^{3} k \frac{v}{\omega} \frac{\eta}{(\eta / 3-1)^{2}+(v / \omega)^{2}} \\
& r_{0} I_{L}(t) \pi r_{0}^{2} t
\end{aligned}
$$

where, $v=\max \left\{\mathrm{v}_{\mathrm{ei}}, \mathrm{v}_{\mathrm{en}}\right\} ; \mathrm{v}_{\mathrm{ei}}=\sqrt{2} \pi \mathrm{Z}^{2} \mathrm{e}^{4} n_{\mathrm{i}} /\left(\mathrm{m}_{\mathrm{e}}{ }^{1 / 2} T_{e}^{3 / 2}\right)$, and $v_{\mathrm{en}} \approx \sqrt{2} \pi \mathrm{Z}^{2} \mathrm{e}^{4} n_{\mathrm{n}} /\left(\mathrm{m}_{\mathrm{e}}{ }^{1 / 2} I_{a}^{3 / 2}\right)$ are electron-ion and electron-atom collision frequencies and $\eta=n_{\mathrm{c}} / n_{\mathrm{c}}, v \approx$ $0.410^{-4} \mathrm{Z} n_{\mathrm{e}} T_{\mathrm{c}}^{3 / 2}$, Plasma temperature has maximum at $\eta=3$ because the absorption coefficient has maximum at the same density. This will occur at the moment when $n_{\mathrm{e}} / n_{\mathrm{c}}=3$ (see Fig. 2):

$$
t_{r} \approx \frac{r_{0}}{c_{s 0}}\left[\left(\frac{Z n_{i 0}}{3 n_{c}}\right)^{1 / 3}-1\right]
$$

Ncxt we estimate the particle lifetime $t_{p 1}$. We suppose that $t_{p}$ is the time taken for the PP density to decrease to the gas density. If we take into account the dependence of $\mathrm{c}_{\mathrm{s}}$ on $T_{e}$, we obtain $\mathrm{t}_{\mathrm{pl}} \approx 0.25\left(n_{0} / n_{\text {air }}\right)$ ${ }^{2 / 3}\left(\mathbf{r}_{0} / \mathrm{c}_{\mathrm{s} 0}\right)$.

As an example, if $\mathrm{r}_{0} \approx 10^{-5} \mathrm{~cm}^{3}, n_{0}=10^{23} \mathrm{~cm}^{-3}, n_{\text {air }}=$ $10^{19} \mathrm{~cm}^{-3}$ and $T_{e} \approx 10 \mathrm{eV}$, then we obtain $\mathrm{t}_{\mathrm{pl}} \approx 1 \mathrm{~ns}$. So laser pulse $\tau_{\mathrm{p}}$ should be close to this time for optimal interaction, but a shorter pulse duration is preferable because for large classical absorption of the laser pulse by PP we should have absorption coefficient $A \approx$ $10\left(v_{\mathrm{e}} / / \omega\right)\left(\mathrm{c}_{\mathrm{s}} \mathrm{t}_{\mathrm{pl}}\right) \approx 1$, and from this condition and our parameters the optimal $\tau_{\mathrm{p}} \approx 0.1 \mathrm{~ns}$. 


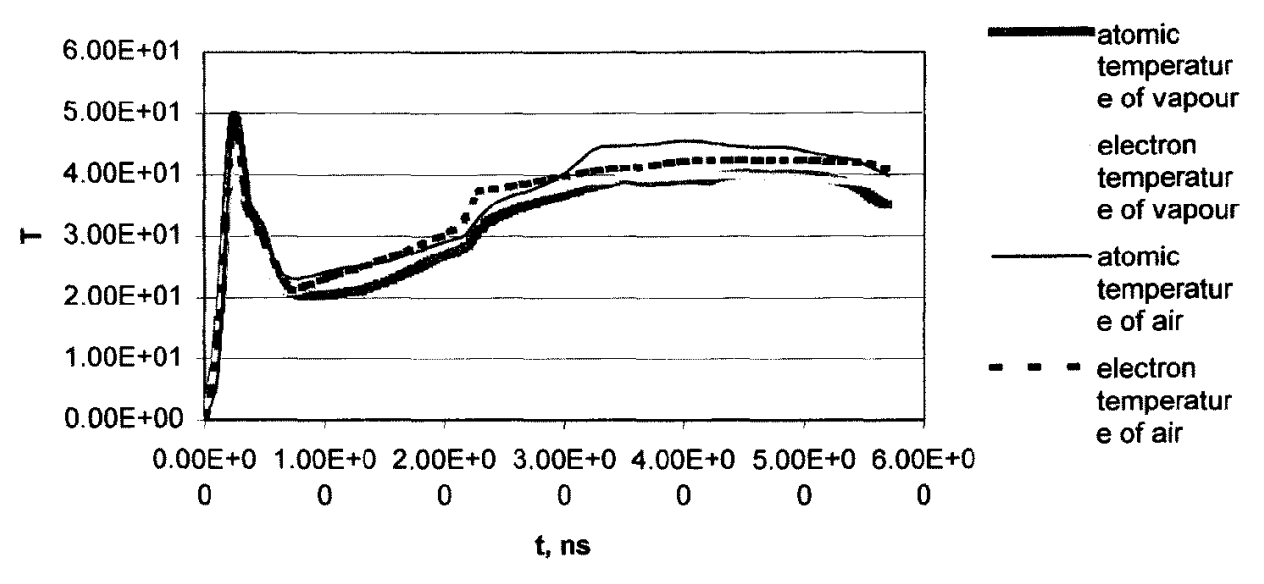

Fig. 2. Time dependence of temperature.

\section{Plasma emission}

As seen from the above, there are two possible variants of SSP size detection by its $\mathrm{PP}$ radiation after LIB.

In the first case at $\mathrm{r}_{0}<\lambda, \tau_{\mathrm{p}}<10 \mathrm{~ns}, \lambda=1 \mu \mathrm{m}$ and $I>10^{11} \mathrm{~W} / \mathrm{cm}^{2}$ the entire focal volume of the gas and particle breaks down and we must consider radiation from this complex area. From the large initial SSP density, its radiation will be more than air radiation initially, but due to the huge volume difference the Bremsstrahlung radiation of air will increase very fast after the radius of $P P$ has doubled. In this case, we should analyze the line emission of elements other than those of air.

In the second case at $\mathrm{r}_{0}<\lambda, \tau_{\mathrm{p}}<10 \mathrm{~ns}$, and $I \approx$ $10^{11} \mathrm{~W} / \mathrm{cm}^{2}$ we can take laser radiation with $\lambda \leq$ $0.5 \mathrm{mkm}$ and air pressure of less than atmospheric pressure because the damage threshold of air increases at $\lambda$ and air pressure decreases as well, thus helping to prevent the breakdown of air instead of SSP. This case was considered in [10]. We therefore focus on the first case and will consider the late stage of the process when emission from SSP plasma exceeds air plasma emission.

To calculate the plasma emission of SSP and gas, we used the code described in section 2. Simulations have shown the following regarding LIB SSP in gas:
- Evaporation through laser irradiation starts from the surface of SSP and the volume increases.

- The evaporated material is quickly ionized if laser intensity exceeds the breakdown threshold.

- The PP absorbs $E_{L}$ while the laser pulse continues and $T$ rises; $T$ then descends slowly after the pulse.

Absorption is decided first by absorption of the solid and at that moment the gas is transparent. The PP size is close to that of SSP, and has resonance. There is sufficient growth in absorption if $T_{\mathrm{e}}$ is high enough.

The surrounding particle gas is then ionized by particle radiation and absorbs much laser light in the regime of "light absorption wave"[1]. Some main factors influence the emission produced by the particle and ambient atmosphere [11], namely, changes in the vaporized amount by plasma shielding, changes in plasma temperature by the absorption of laser radiation, and changes in the plasma expansion during and after the laser pulse. The increase in the emission intensities in the rarefied atmosphere is related to the first factor. As to the second factor, the plasma temperature is higher for denser conditions. In addition to the higher excitation temperature, the slower decay rate of the temperature is also important, which is related to the third factor. Plasma confinement by surrounding atmosphere prevents fast expansion of plasma. Gas breakdown decreases the amount of vaporized sample. The calculated maximum in the line emission intensity when it changes as a function of the ambient pressure, is explained by these mechanisms. The differences in emitted intensities in $\operatorname{Ar}(\boldsymbol{Q})$ and air ( $O$ ) can be explained by the difference in its densities and thermal conductivities (see Fig. 3). 


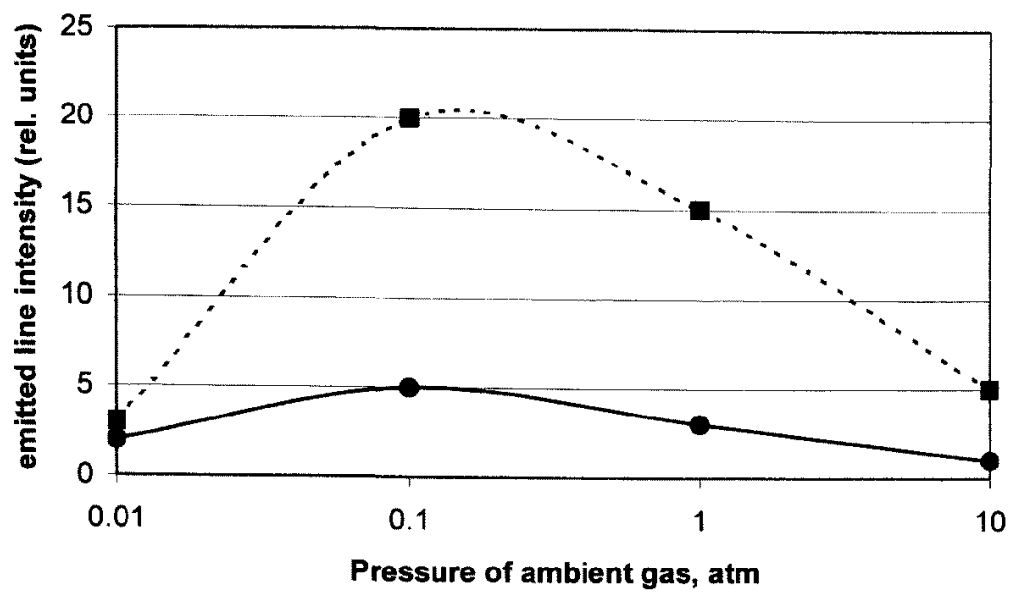

Fig. 3. Dependence of emitted line intensity on pressure of ambient gas.

Figure 4 shows the dependence of rate $K_{r}=E_{r} / E_{a}$ on initial particle size $r_{0}$, where $E_{r}$ is total energy emitted in the line spectral range $[4.7,5.1 \mathrm{eV}]$ and integrated in space at the moment $\mathrm{t}=100$ ns divided by laser absorbed energy $E_{a}$. This dependence is approximately linear and allows us to determine the particle size from the plasma emission after LIB.

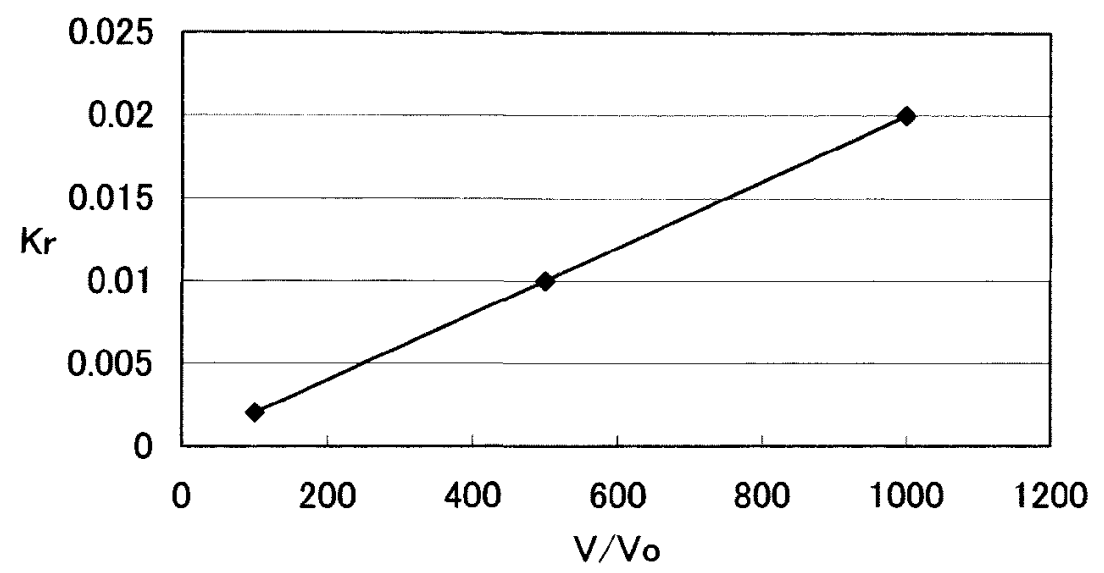

Fig. 4. Dependence of $\mathrm{K}_{r}=\mathrm{E}_{\mathrm{r}} / \mathrm{E}_{\overline{\mathrm{a}}}$ on initial particle volume

\section{Conclusion}

We developed an analytical model and simulation code to calculate laser pulse interaction with a small solid particle in a gas atmosphere. The temporal dynamics of plasma parameters showed a large rise of electron temperature and emitted light at the moment of resonant conditions, even for small laser intensity. At that moment, the surrounding particle gas was ionized by particle plasma radiation and absorbed much laser light. Thereafter, the total light emission from air exceeds the emission from the plasma particle from the big gas radiated volume, but the line emission from particle plasma permits detection of the target material at a late stage of the process.

The increased dependence of SSP plasma line emission rate on initial particle size permits this method to be used for measuring particle size for particle diameters of less than $0.1 \mu \mathrm{m}$.
This work was supported by the R\&D Institute for Photonics Engineering, the Manufacturing Science and Technology Center, and the New Energy and Industrial Technology Development Organization.

(Manuscript received May 14, 2001) 


\section{References}

1. Yu. P. Raizer, Laser-induced discharge phenomena: "Studies in Soviet Science," NY, 1977.

2. T. Kitamori, K. Yokose, K. Suzuki, and T. Sawada, Jpn. J. Applied Physics, 27, No. 6, pp. L983-L985, 1988, S.I. Anisimov, Sov. Phys. JETP, 27, p. 182, 1968.

3. G.H. Canavan and P.E. Nielsen, Appl. Phys. Lett., 22, p. 40, 1973.

4. D.C. Smith, Journal of Applied Physics, 48, No. 6, p. 2217, 1977.

5. T. Ueda, H. Hayashi, A.A. Andreev, M. Wakamatsu, H. Kudou, and T. Sugiyama, The 4th Symposium on Advanced Photon Processing and Measurement Technologies, p. 142, 2000.

6. A.A. Andreev, A.N. Semakhin, and V.V. Akulinichev, Probl. Nauchn. Priborostr. 3, 8841993.

7. A.A. Samarskii and V.G. Popov, "Numerical schemes of gas dynamics," M., 1975.

8. A.P. Golub, Journal Calculation Math. and Math. Phys., vol. 23, p. 142-151, 1983.

9. A. Andreev, T. Ueda, and M. Wakamatsu, Proceedings of SPIE vol. 3935 , p. $139,2000$.

10. A. Andreev, T. Ueda, M. Wakamatsu, and H. Hayashi, ECLIM, Prague, June 2000.

11. Y. Iida, Spectrochimica Acta, vol. 45B, p. 1353, 1990.

12. I.B. Kosyrev et al., Astronom. Vestnik, vol. 30, p. 316, 1996.

\section{Alexander Andreev (Non-member)}

Alexander Andreev was born in Moscow,

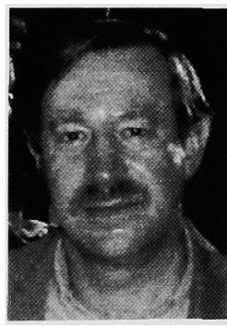

Russia, on November 11, 1949. He received the B.E. and M.E. degree in physics from Leningrad State University, Leningrad, Russia, in 1970 and 1972 respectively. He received $\mathrm{Ph}$.D degree from the same University in 1979. He received Doctor of Science degree from Vavilov State Optical Institute, St. Petersburg, Russia in 1991. In 1993 he got the position of professor from State Technical University of St. Petersburg. Since joining Vavilov State Optical Institute in 1972 he has been engaged in research and development of high power lasers and their applications. Now he is a chief of theoretical laboratory in Institute of Laser Physics, St. Petersburg, Russia. He is co-author of more than 100 publications and two books. Prof. Andreev is a member of ROS, SPIE and OSA.
Toshitsugu Ueda (member)

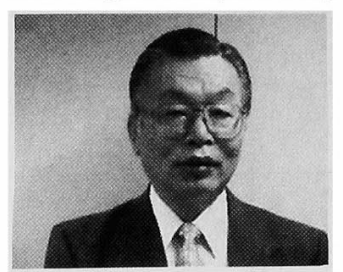

Toshitsugu Ueda was born in Nara, Japan, on October 4, 1945. He received the B.E. and M.E. degree in electrical engineering from Shinshu University, Nagano, Japan, in 1969 and 1971 respectively. He received $\mathrm{Ph} . \mathrm{D}$. degree from Tokyo Institute of Technology in 1988.

Since joining Yokogawa Electric Corporation in 1971, he has been engaged in developing low noise amplifiers, mechanical resonators, micro machining technologies and sensors using above mentioned technologies for temperature, pressure and displacement. Now he is a general manager of Corporate R\&D department.

He received Awards from Society of Instrument and Control Engineers of Japan in 1987 and 1994, and Awards from Japan Institute Invention and Innovation in 1985 and 1987. Dr. Ueda is a member of the Institute of Electrical Engineers of Japan, and Society of Instrument and Control Engineers of Japan. 